\title{
Language, Music, and Brain
}

\section{Mehdi Madanifard"}

Department of Cognitive Linguistics, Tarbiat Modares University, Tehran, Iran

\section{A BSTRACT}

Introduction: Over the last centuries, scientists have been trying to figure out how the brain is learning the language. By 1980, the study of brain-language relationships was based on the study of human brain damage. But since 1980, neuroscience methods have greatly improved. There is controversy about where music, composition, or the perception of language and music are in the brain, or whether each of the cerebral hemispheres plays a role in language and music processes and despite the many discoveries and findings, there is still no definite answer. From the very beginning, the child is able to hear the sounds. These sounds are initially raw and pointless and gradually begin with the child's interaction with the language learning environment. Language like music has powerful rhythm patterns. The syllable timing helps the listener to recognize a sound from another and to understand what the other says. The ability to recognize the difference in sounds helps babies to speak. Many studies have suggested that the processing and perceptual regions of music overlap with the areas involved in comprehension and linguistic comprehension. Furthermore, there is a positive relationship between the practice and skill in musical and cognitive abilities, such as language. Longterm reinforcement of this relationship creates new cognitive abilities other than music. Conclusion: Music stimulates many areas in the brain in addition to the cerebral cortex, Key words: which overlap with many linguistic processing areas, such as the left temporal region. The syntax of musical sentences is processed in the same regions where the syntax of linguistic sentences is processed.

3. Brain

*Corresponding Author: Mehdi Madanifard

E-mail: psy.madani@yahoo.com 


\section{زبان، موسيقى و مغز}

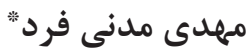

كروه زبان شناسى شناختى، دانشَاه تربيت مدرس، تهران، ايران

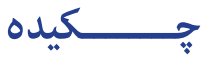

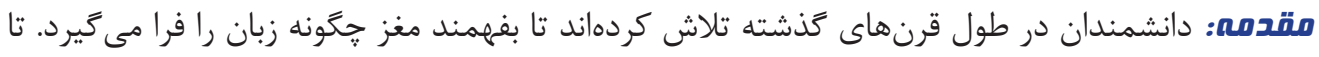

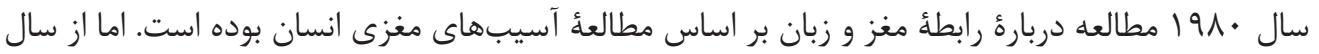

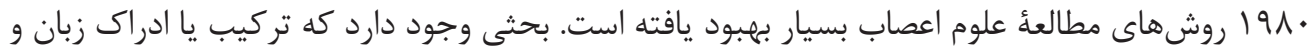

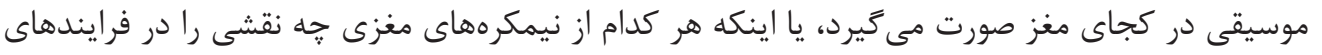

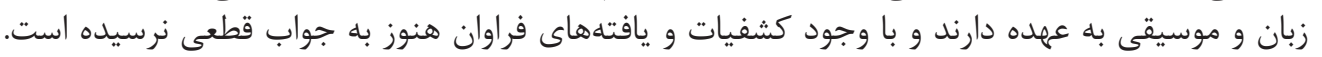

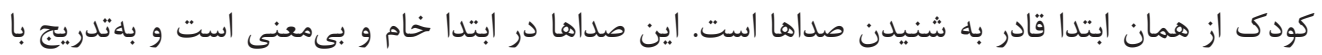

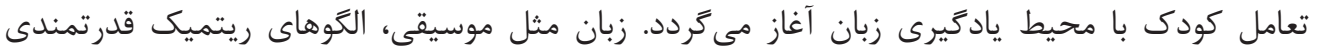

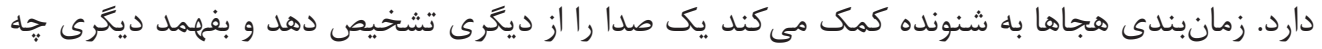

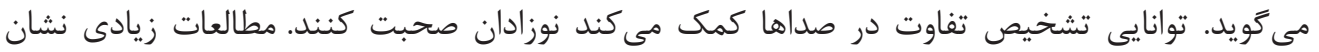

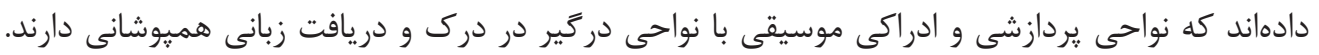

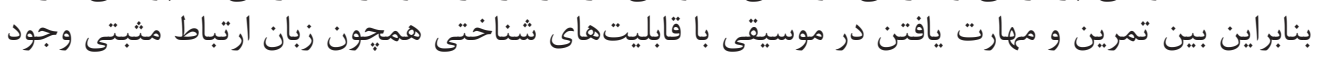

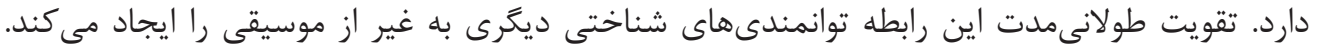

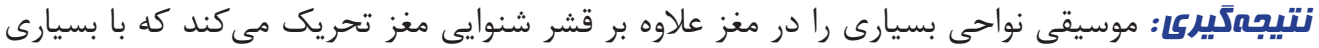

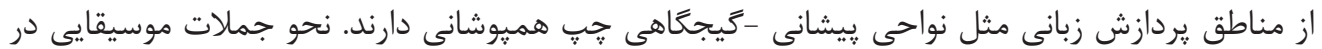

كليد وازهها:

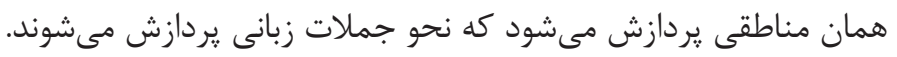




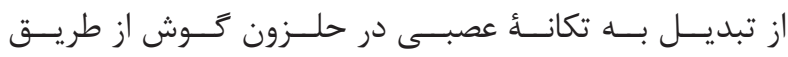

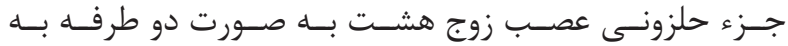

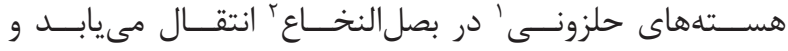

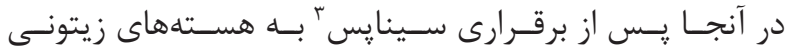

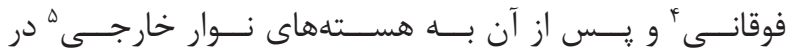

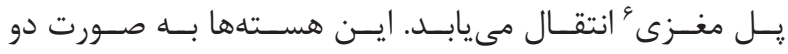

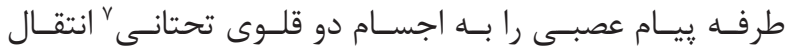

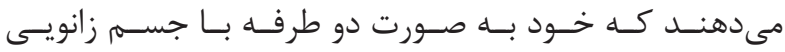

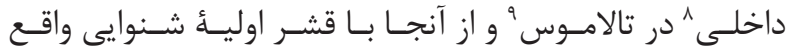

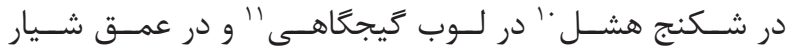

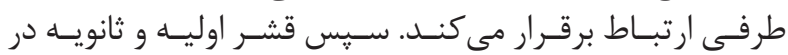

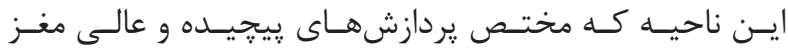

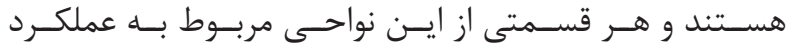

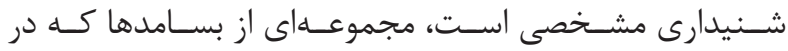

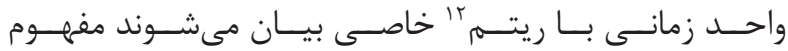

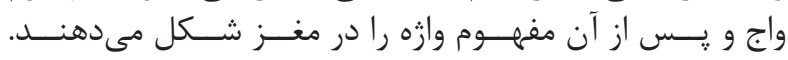

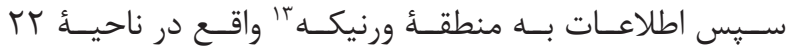

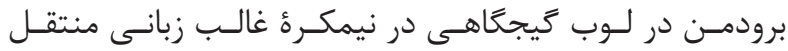

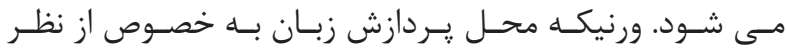

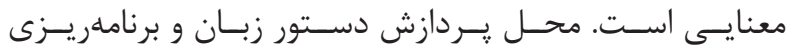

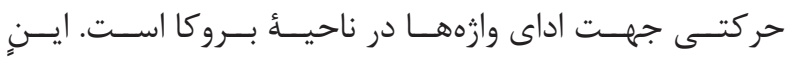

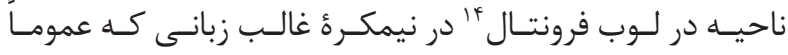

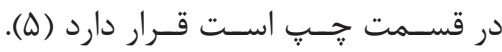

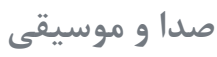

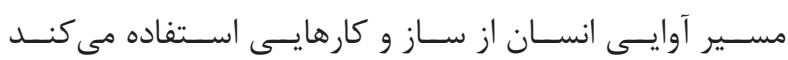

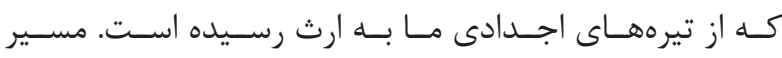

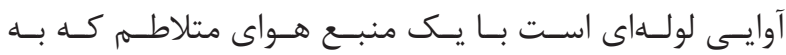

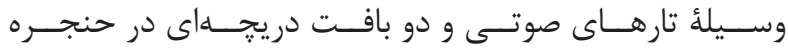

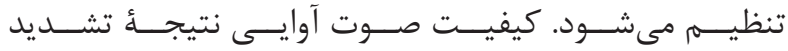

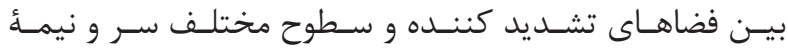

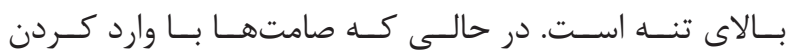

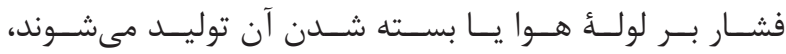

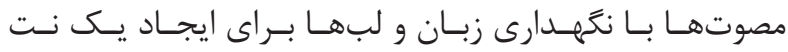

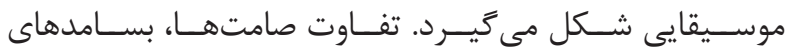

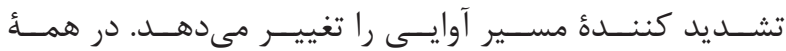

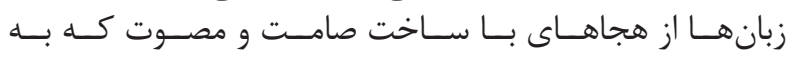

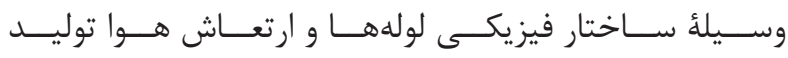

مىشــود، اســتفاده مى گَــردد (؟).

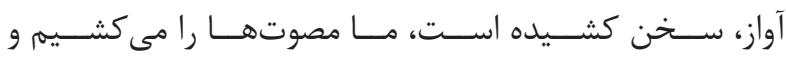

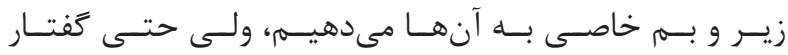

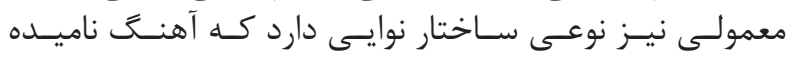

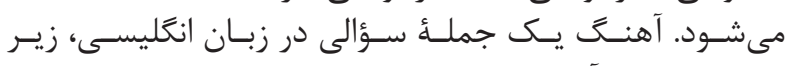

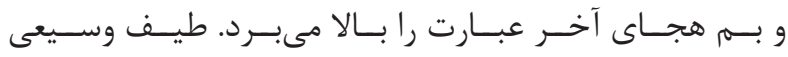

${ }^{1}$ Nuclei cochleares

${ }^{2}$ Medulla oblongata

${ }^{3}$ Synapse

${ }^{4}$ Olivary nucleus

${ }^{5}$ Columnae posterioris lemniscique medialis

${ }^{6}$ Pons

${ }^{7}$ Inferior culliculus

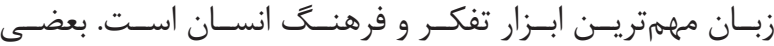

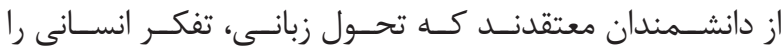

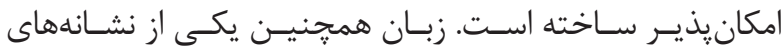

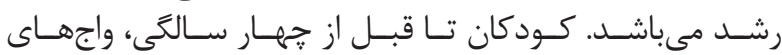

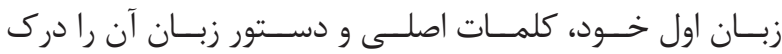

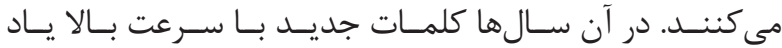

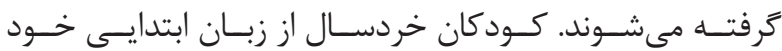

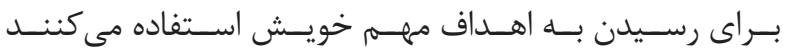

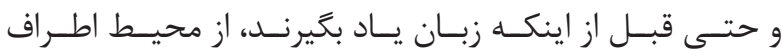

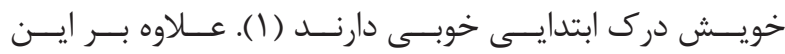

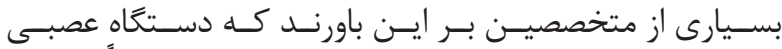

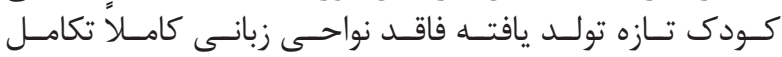

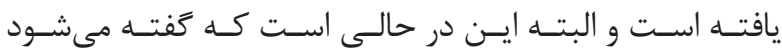

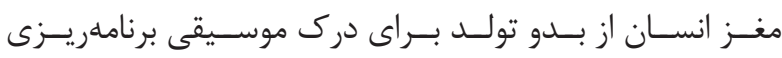

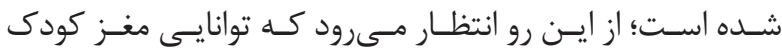

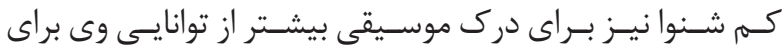

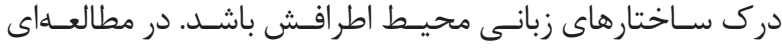

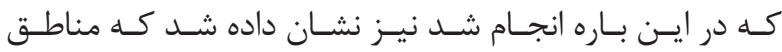

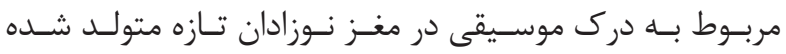

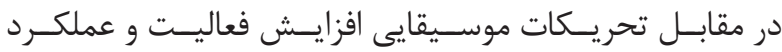

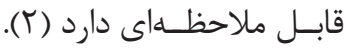

مبانى زيستى زبان

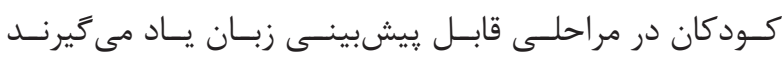

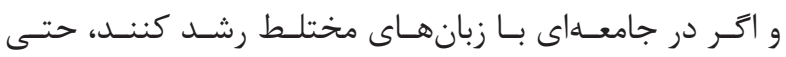

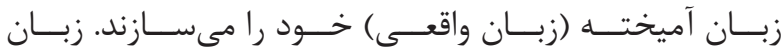

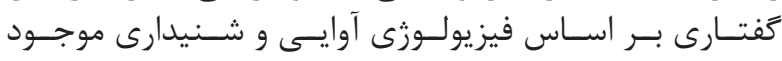

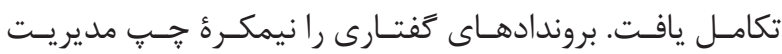

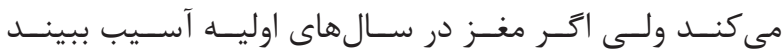

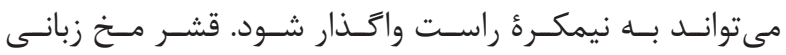

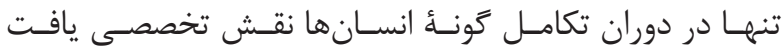

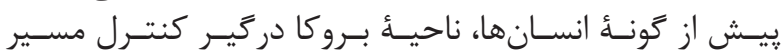

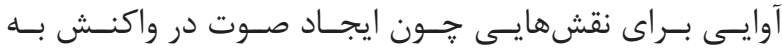

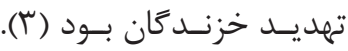

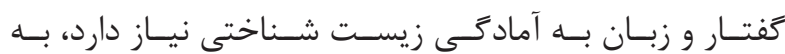

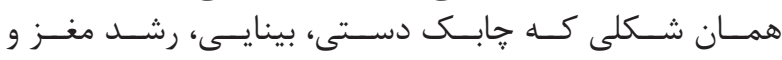

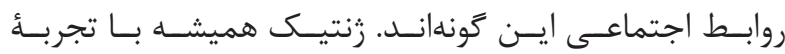

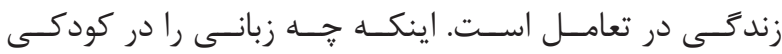

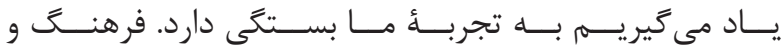

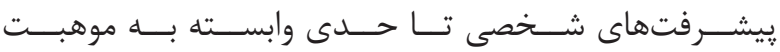

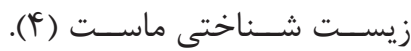

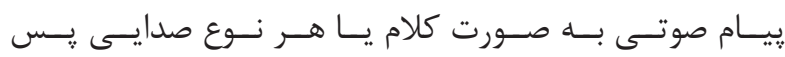

${ }^{8}$ Media geniculate body

${ }^{9}$ Thalamus

${ }^{10}$ Heschl

${ }^{11}$ Temporal lobe

${ }^{12}$ Rhythm

${ }^{13}$ Wernicke

${ }^{14}$ Frontal lobe 
فرهنخَهــاى مختلـف، انـواع مختلفـى از موســيقى دارنـد، بــهـ

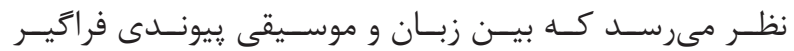

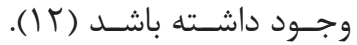

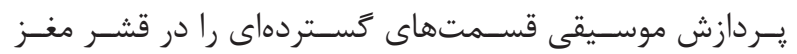

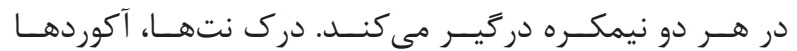

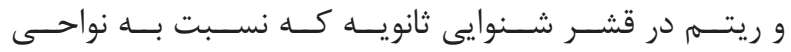

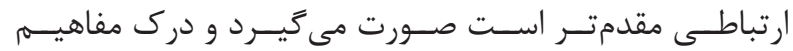

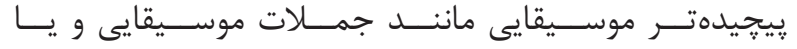

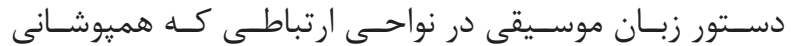

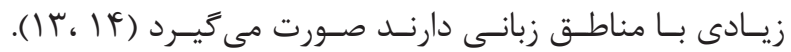

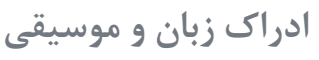

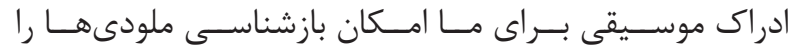

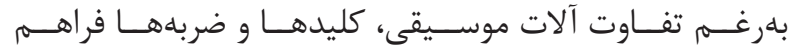

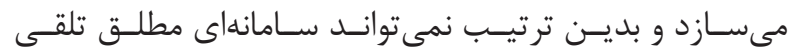

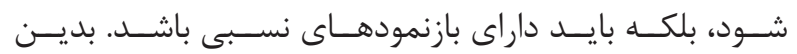

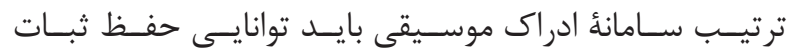

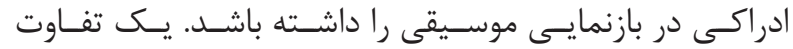

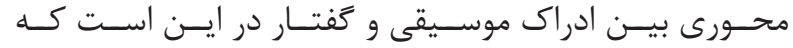

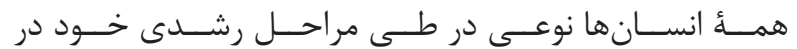

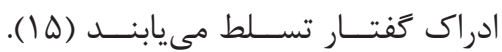

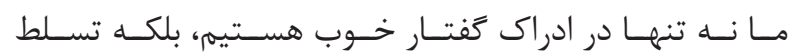

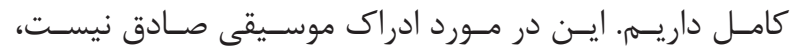

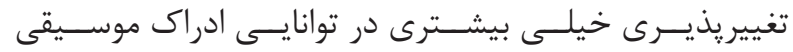

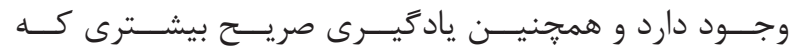

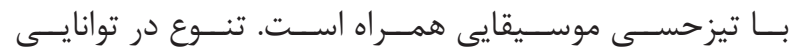

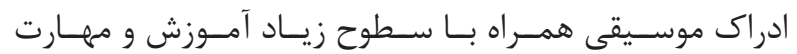

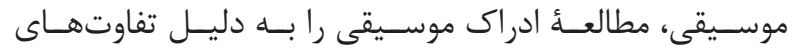

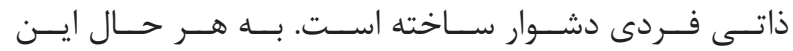

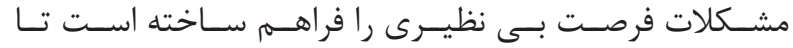

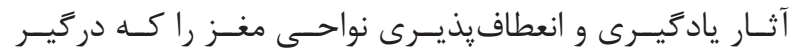

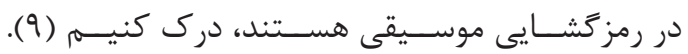

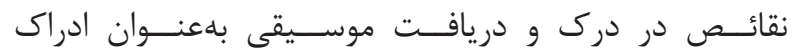

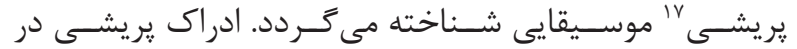

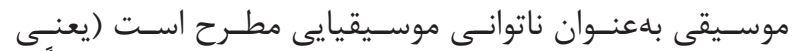

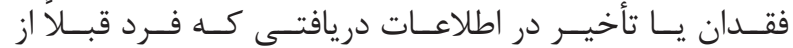

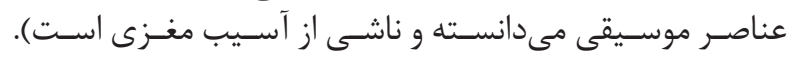

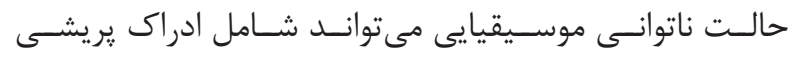

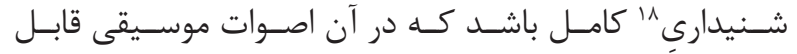

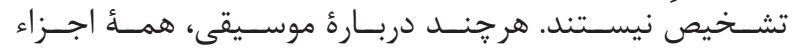

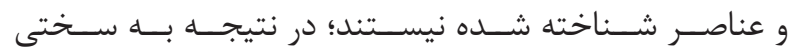

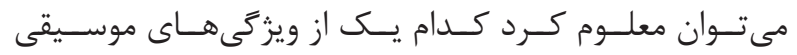

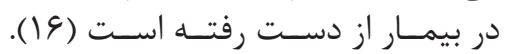

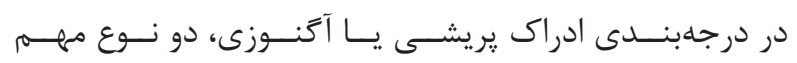

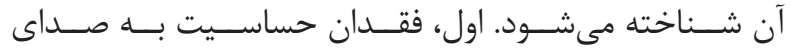

\section{${ }^{15}$ Octave}

${ }^{16}$ Twang

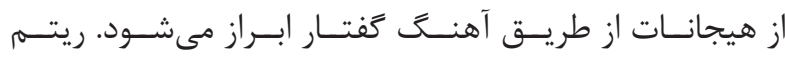

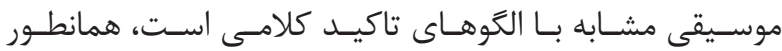

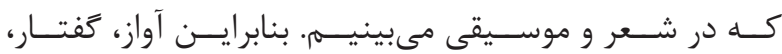

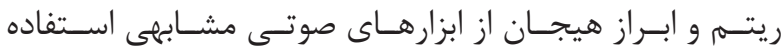

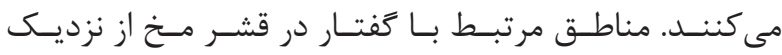

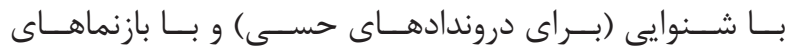

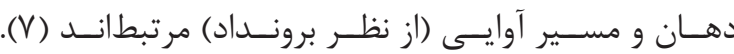

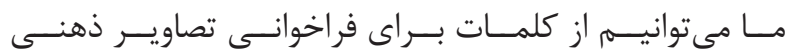

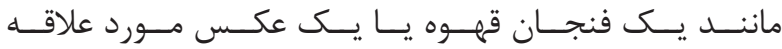

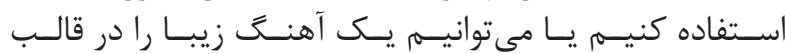

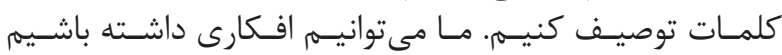

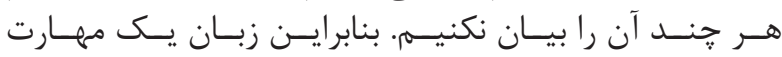

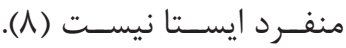

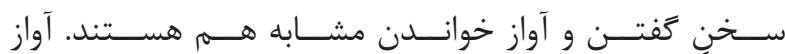

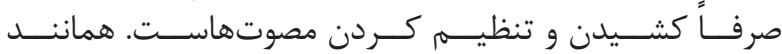

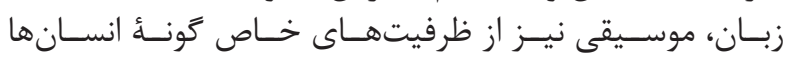

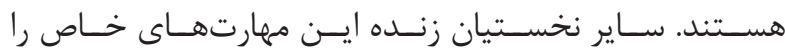

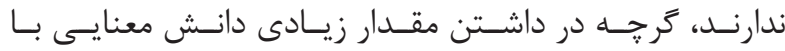

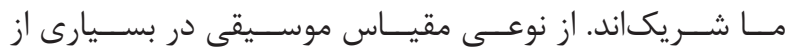

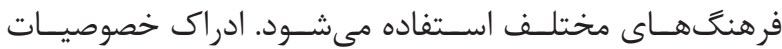

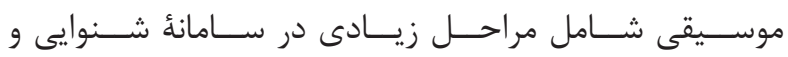

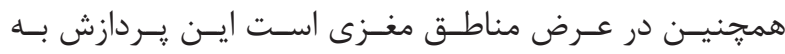

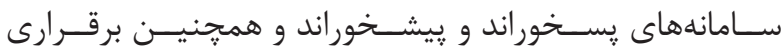

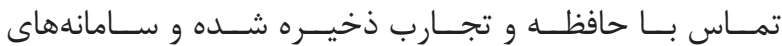

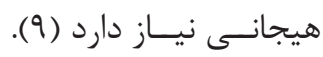

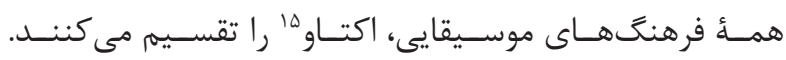

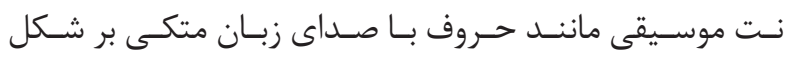

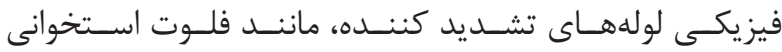

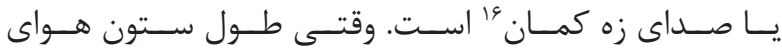

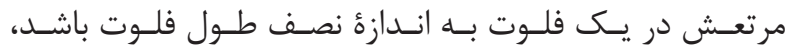

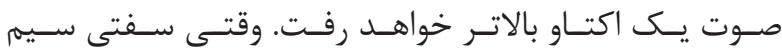

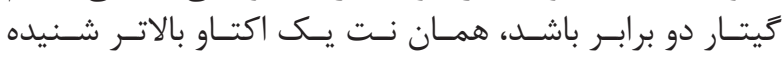

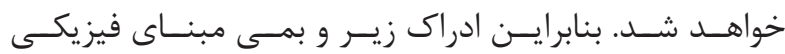

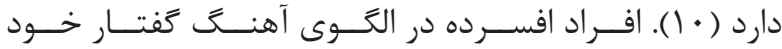

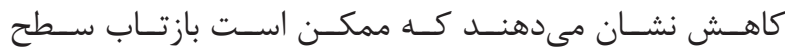

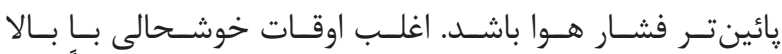

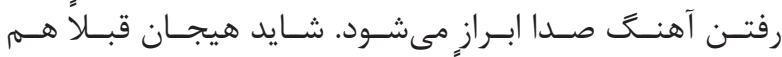

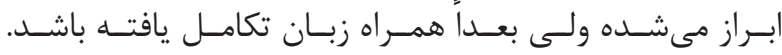

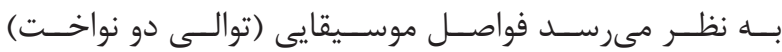

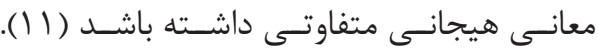

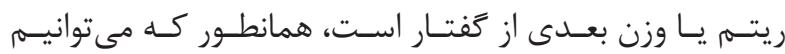

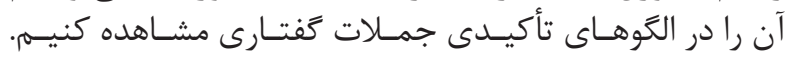

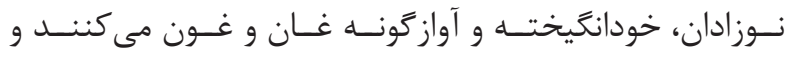

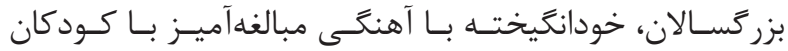

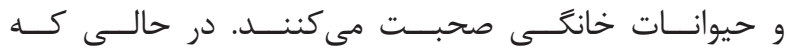

${ }^{18}$ Auditory agnosia 


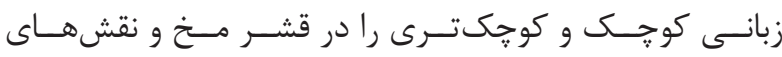

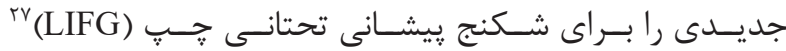
كشـف مى كردمانســـ (9). الكوهاى نيمكر هاى ادراى زبان و موسيقى

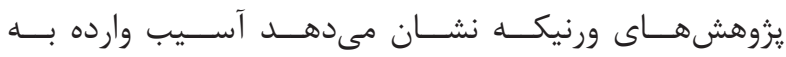

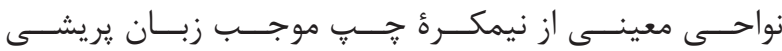

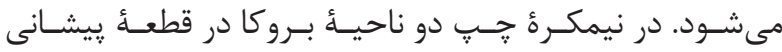

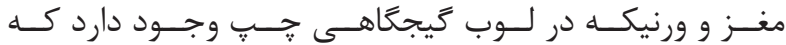

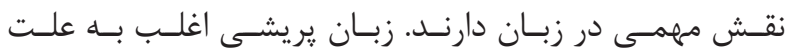

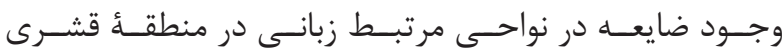

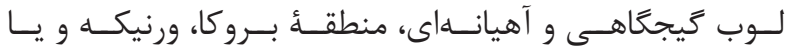

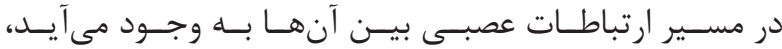

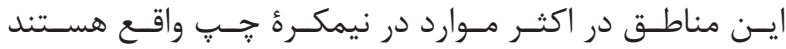

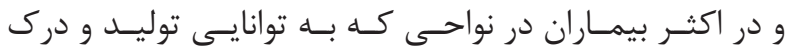

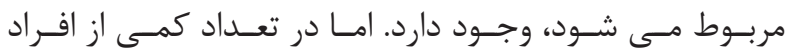

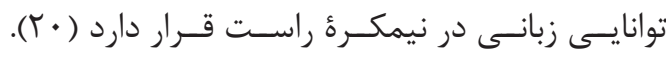

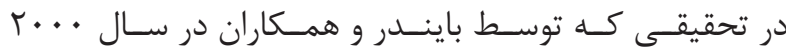

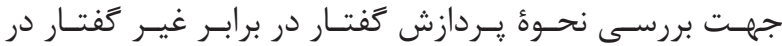

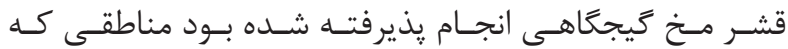

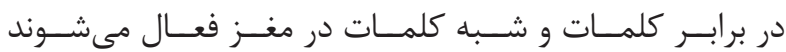

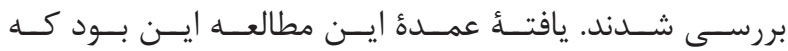

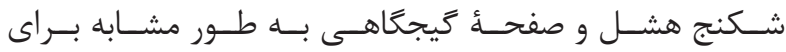

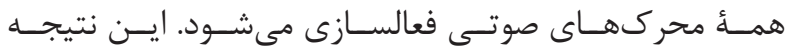

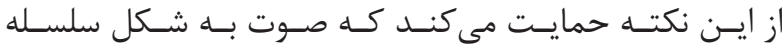

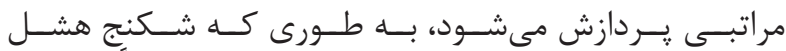

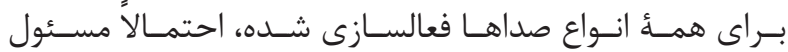

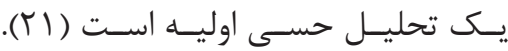

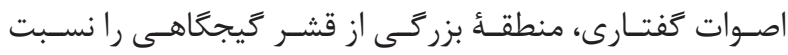

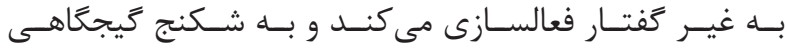

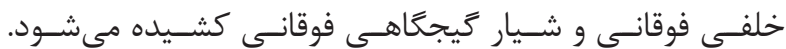

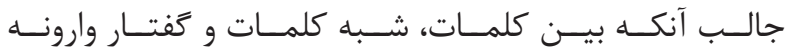

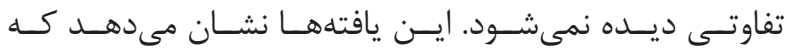

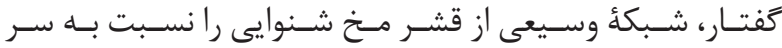

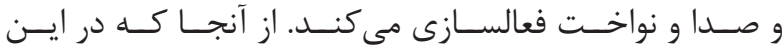

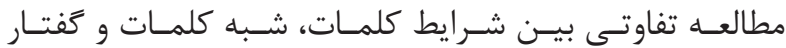

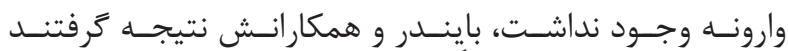

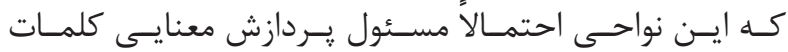

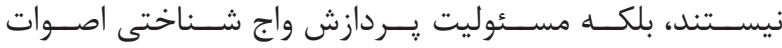

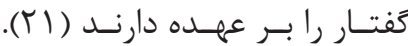

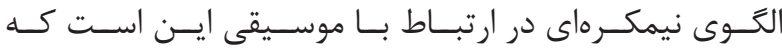

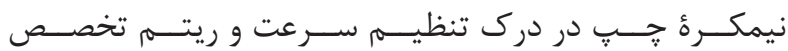

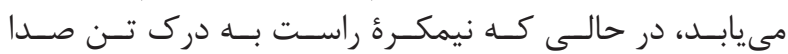

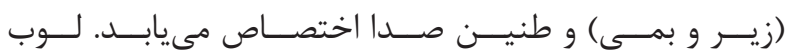

${ }^{19}$ Amelodia

${ }^{20}$ Milner

${ }^{21}$ Seashor

${ }^{22}$ Wanger

${ }^{23}$ Aphasia

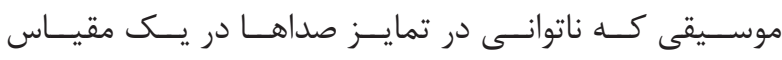

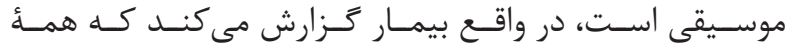

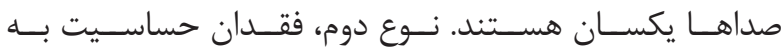

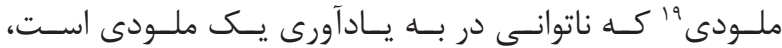

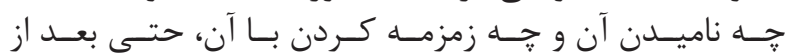

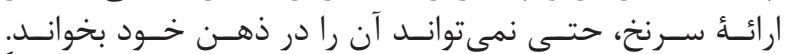

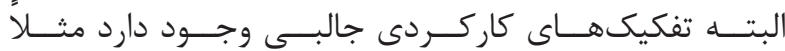

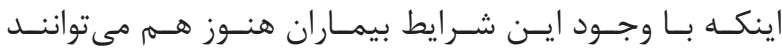

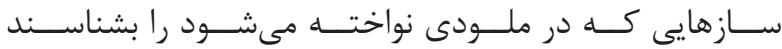

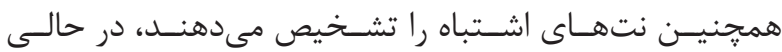

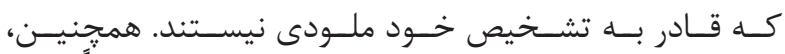

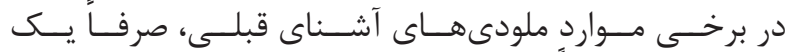

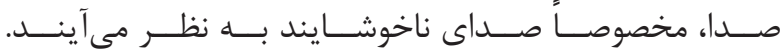

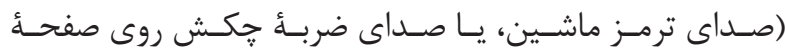

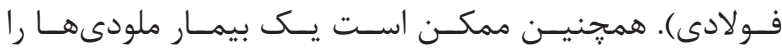

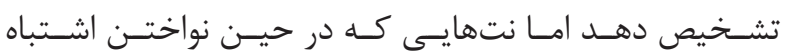

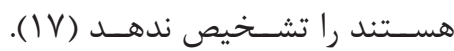

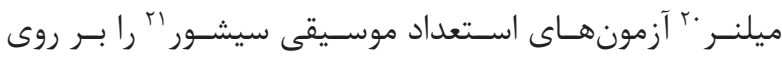

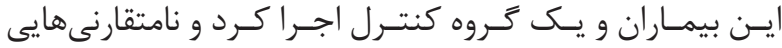

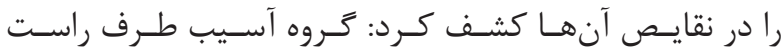

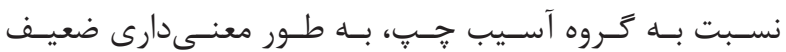

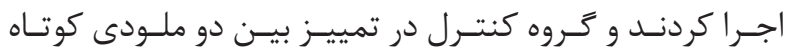

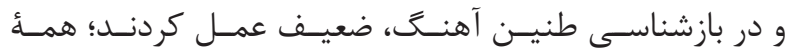

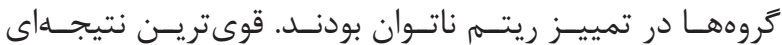

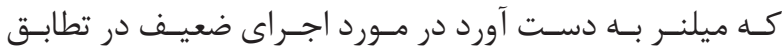

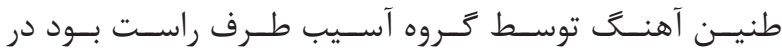

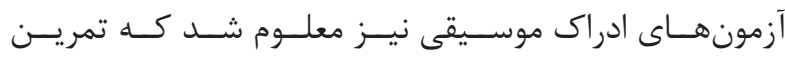

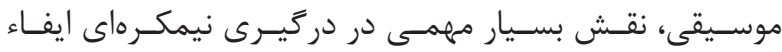

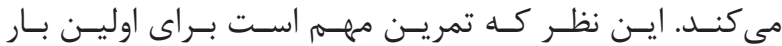

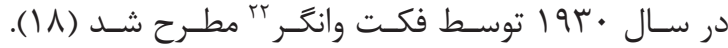

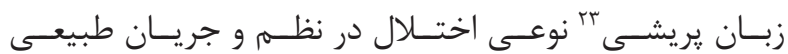

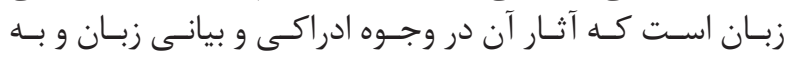

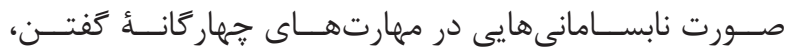

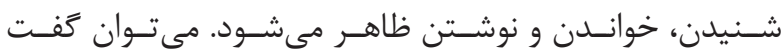

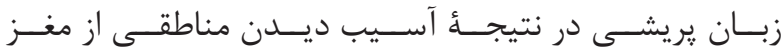

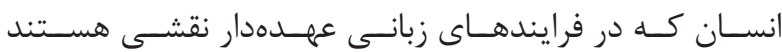

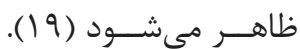

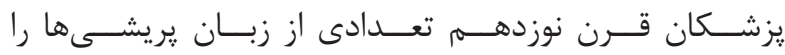

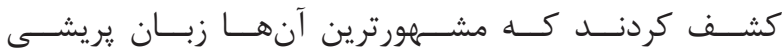

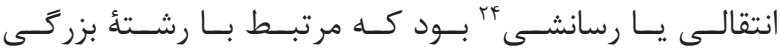

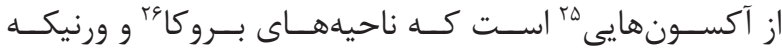

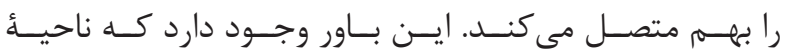

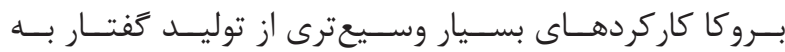

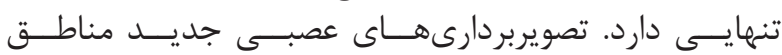

${ }^{24}$ Amnestic aphasia

${ }^{25}$ Axon

${ }^{26}$ Broca

${ }^{27}$ Left Inferior frontal gyrus 


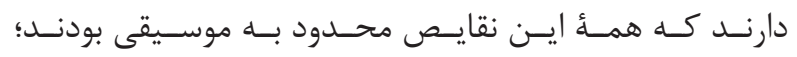

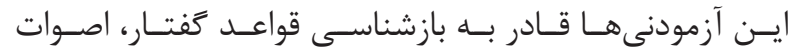

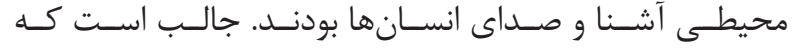

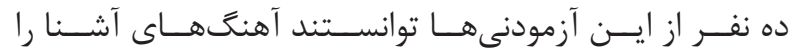

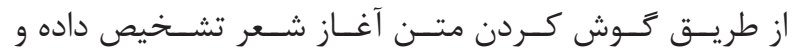

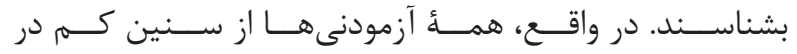

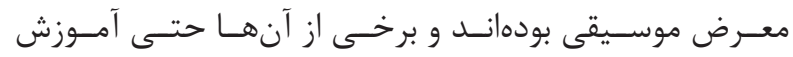

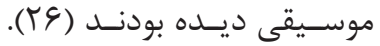

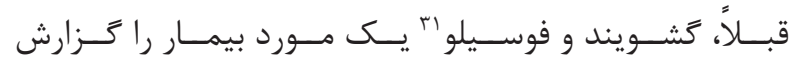

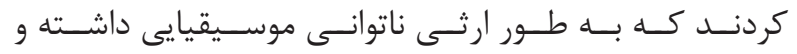

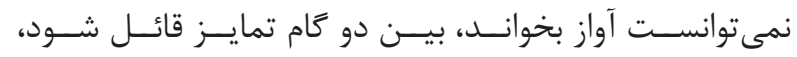

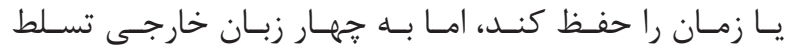

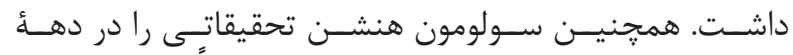

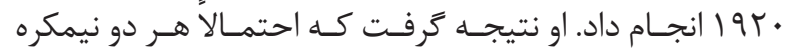

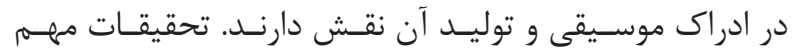

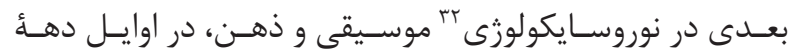

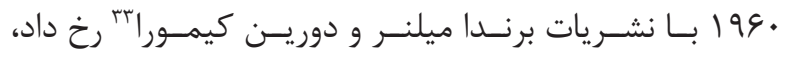

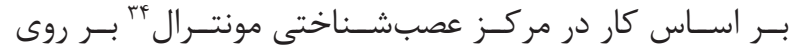

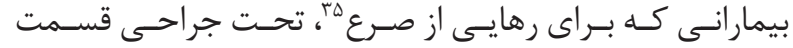

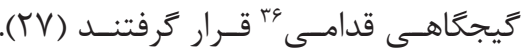

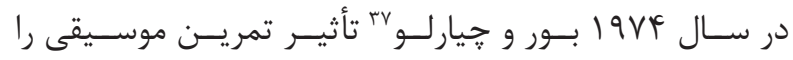

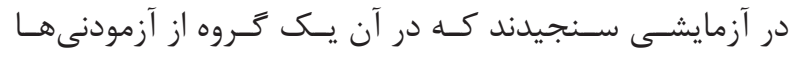

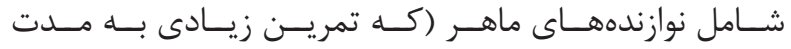

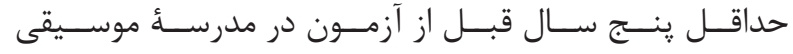

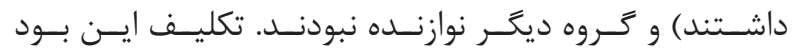

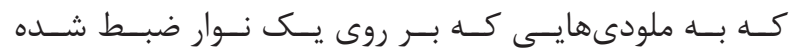

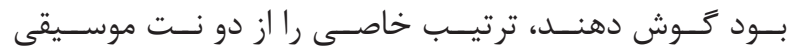

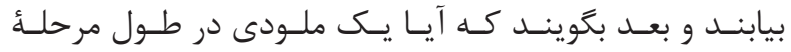

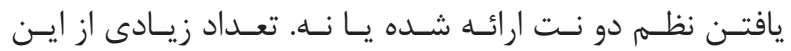

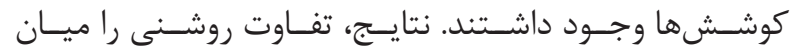

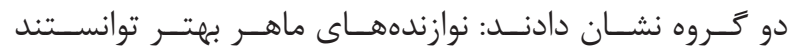

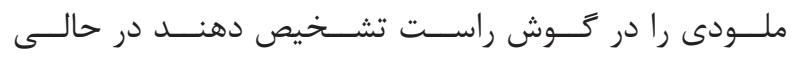

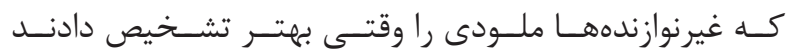

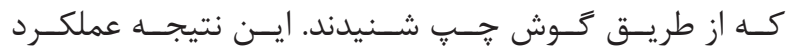

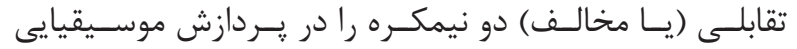

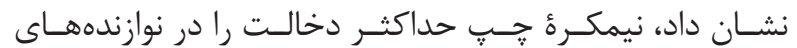

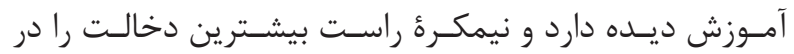

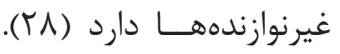

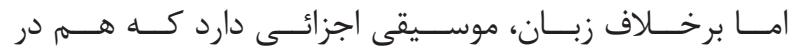

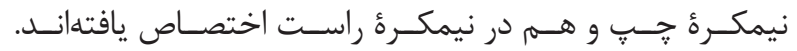

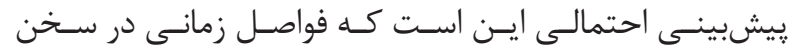

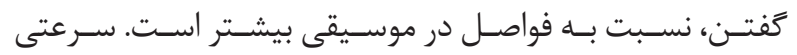

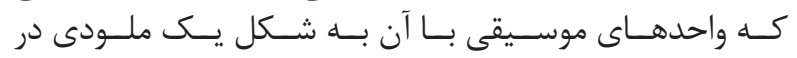

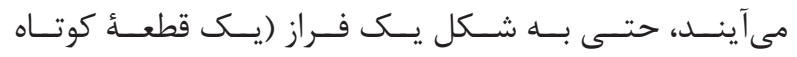

${ }^{28}$ Temporal lobe

${ }^{29}$ Broca aphasia

${ }^{30}$ Amusia

${ }^{31}$ Fosillo and Geschwind

${ }^{32}$ Neuropsychology

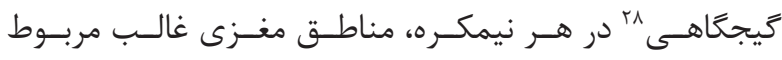

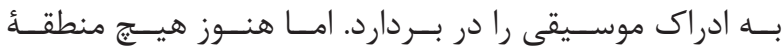

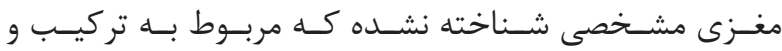

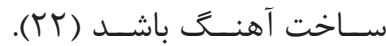

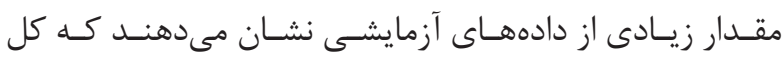

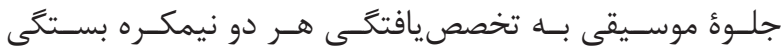

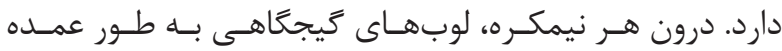

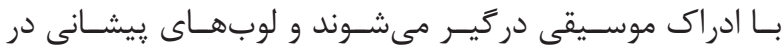

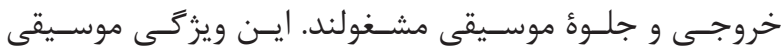

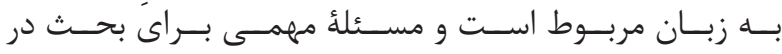

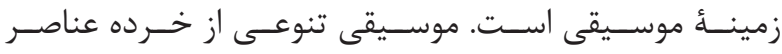

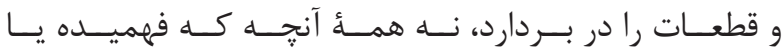

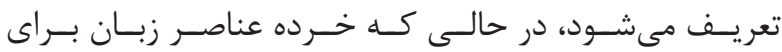

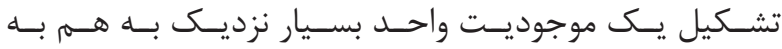

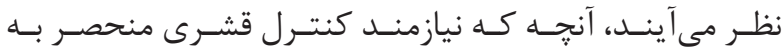

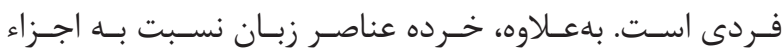

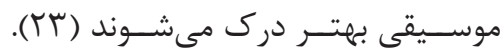

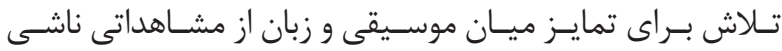

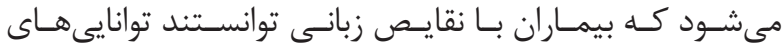

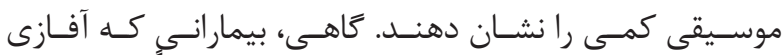

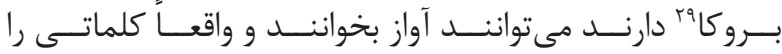

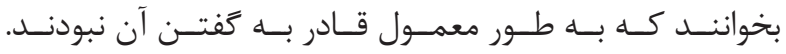

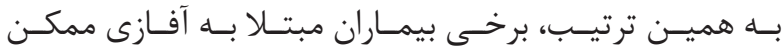

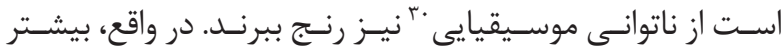

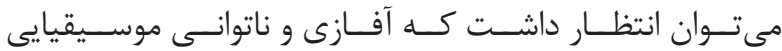
ناشـى از صدمـــ نيمكــرهُ جـــ

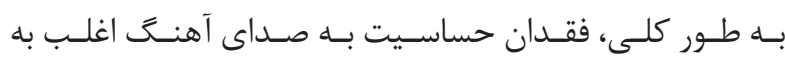

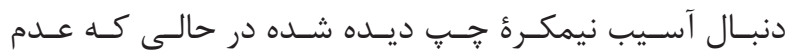

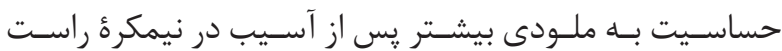

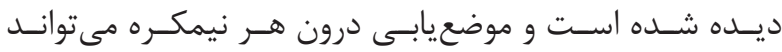

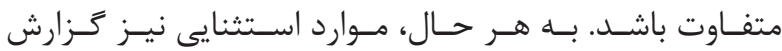

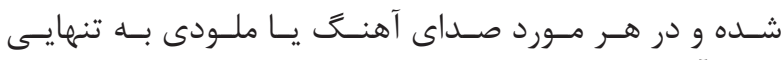

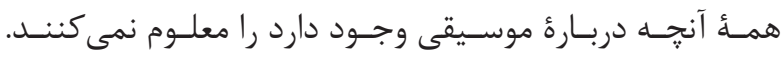

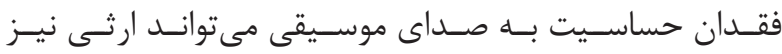

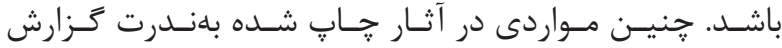

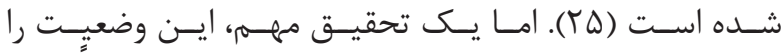

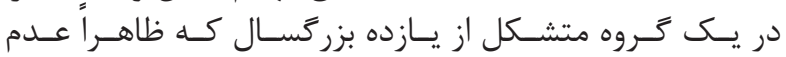

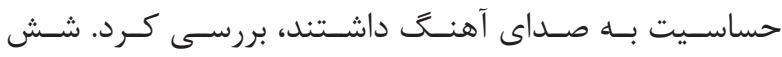

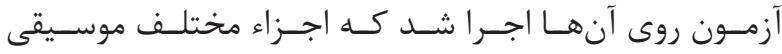

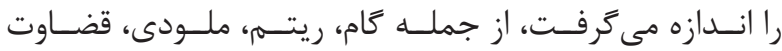

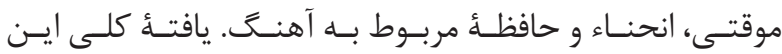

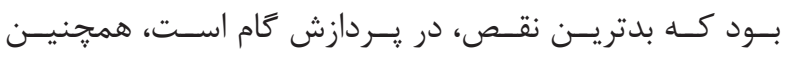

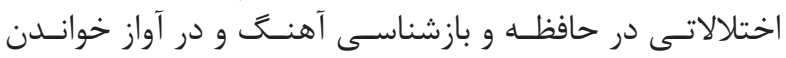

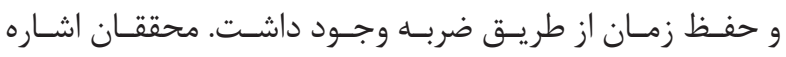

\footnotetext{
${ }^{33}$ Kimura

${ }^{34}$ Montreal neurological center

${ }^{35}$ Epilepsy

${ }^{36}$ Anterior temporal

${ }^{37}$ Bour and Chiarloo
} 


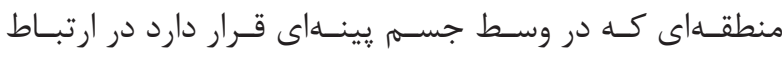

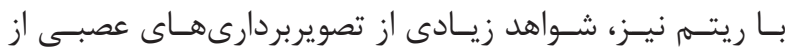

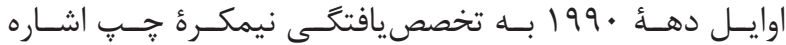

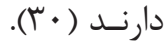

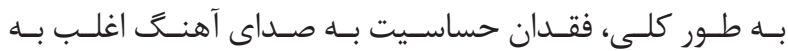

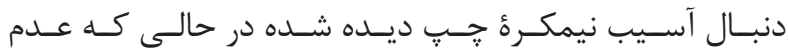

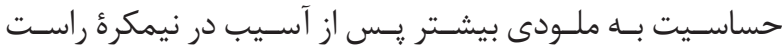

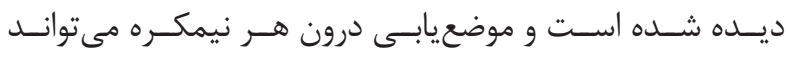

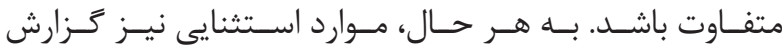

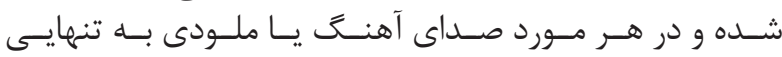

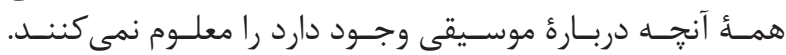

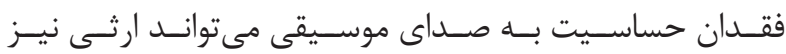

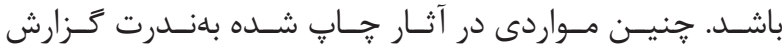

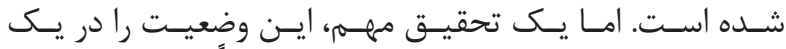

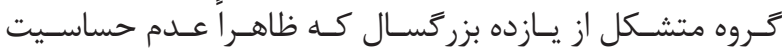

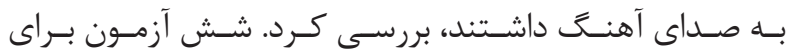

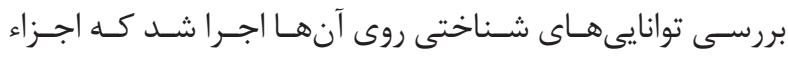

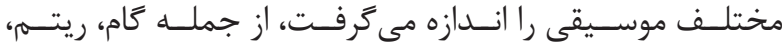

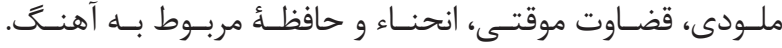

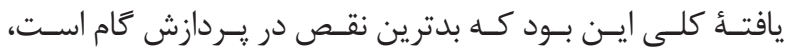

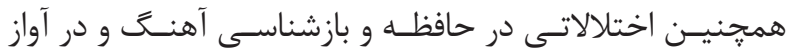

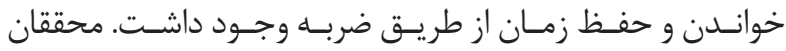

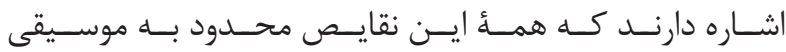

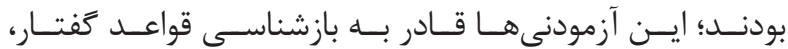

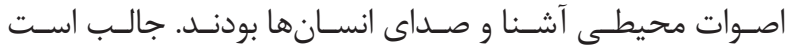

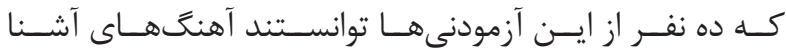

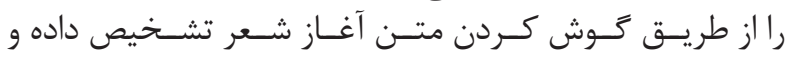

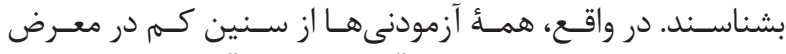

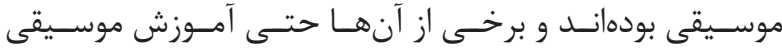

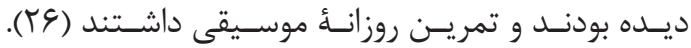

نتيجه گيرى

ادراك خصوصيـات موسـيقى شـامل مراحــل زيــادى يــردازش

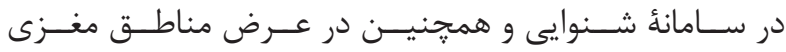

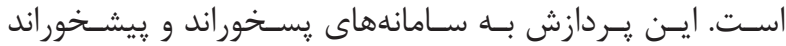

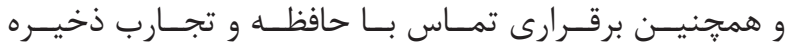

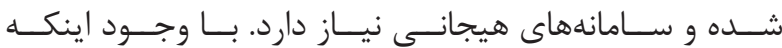

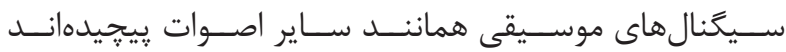

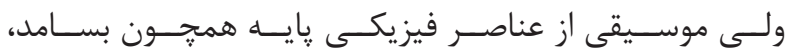

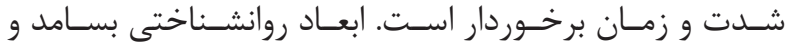

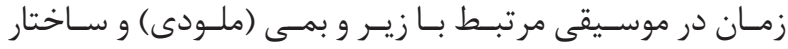

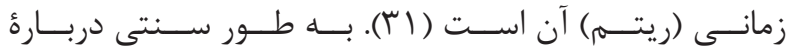

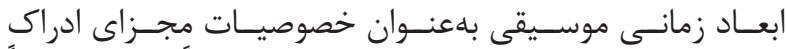

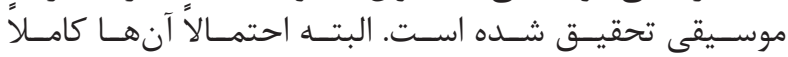

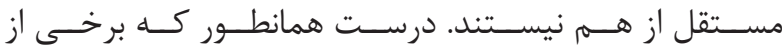

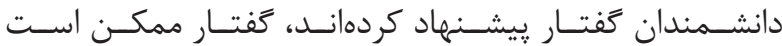

\footnotetext{
${ }^{38}$ Neuroimaging

${ }^{39}$ Cognition information

${ }^{40}$ Right frontal cortex
}

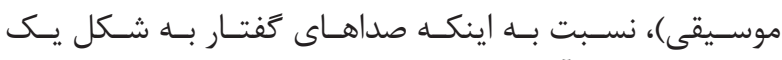

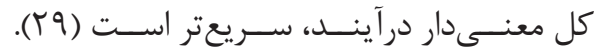

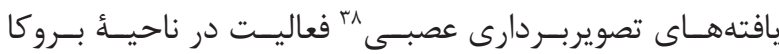

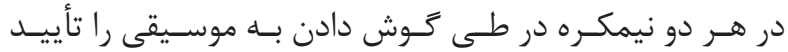

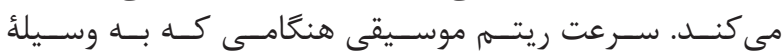

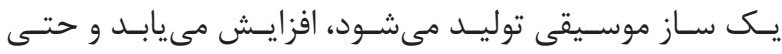

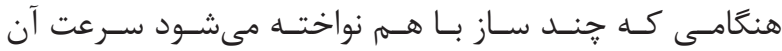

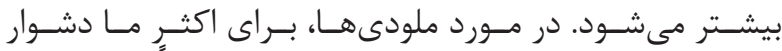

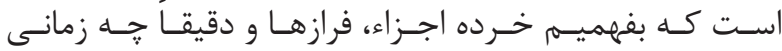

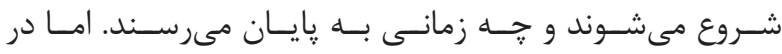

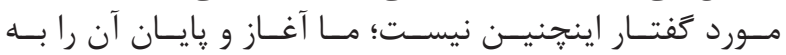

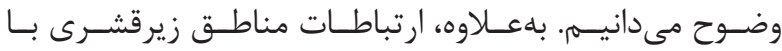

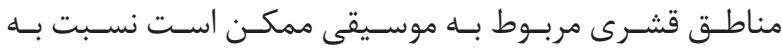

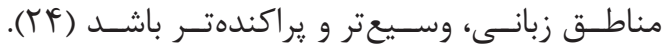

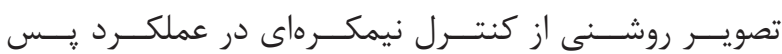

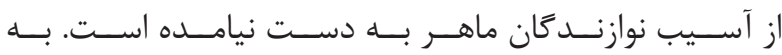

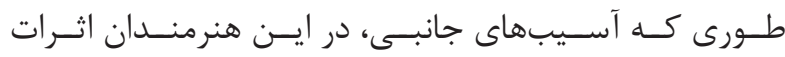

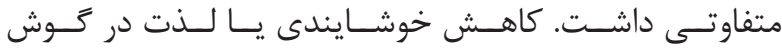

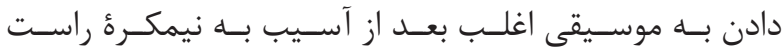

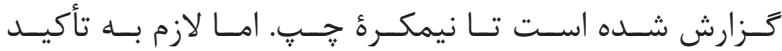

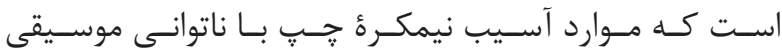

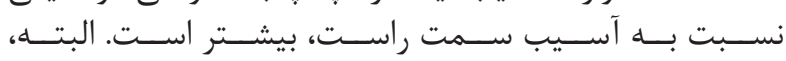

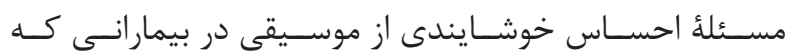

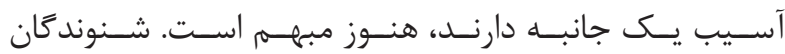

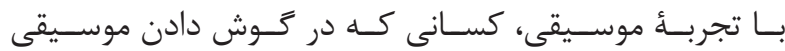

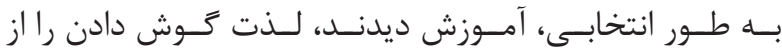

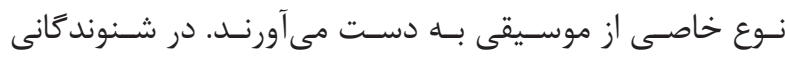

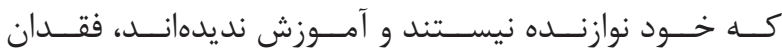

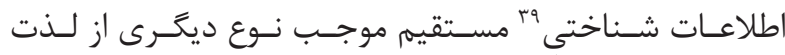

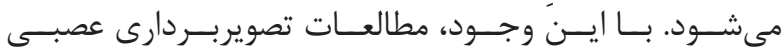

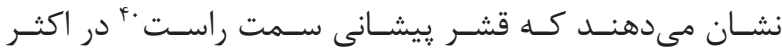

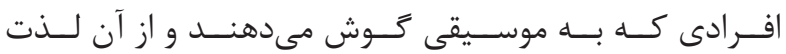

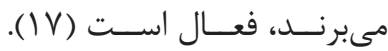

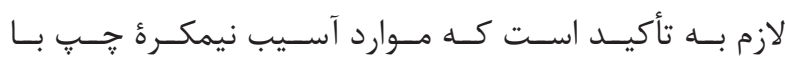

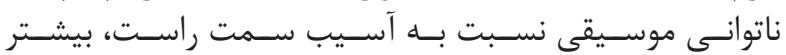

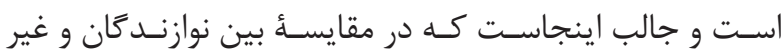

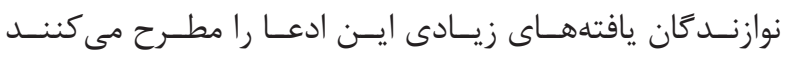

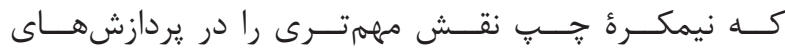

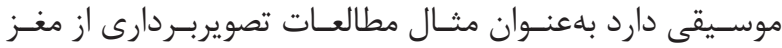

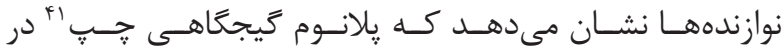

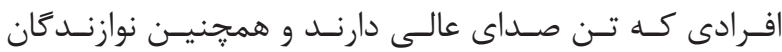

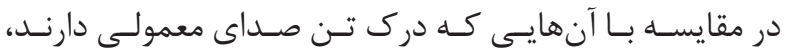

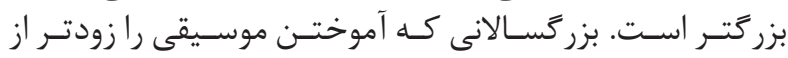

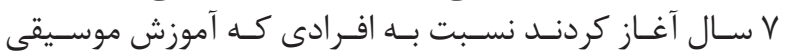

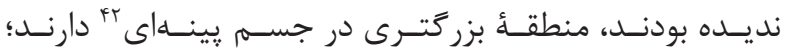

${ }^{41}$ Left planum temporal

${ }^{42}$ Corpus callosum 


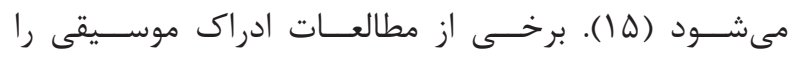

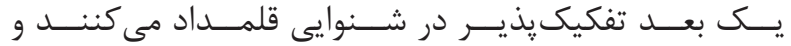

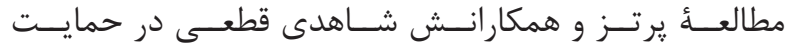

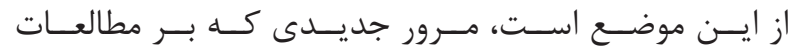

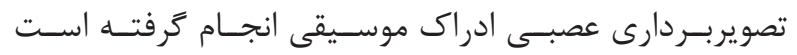

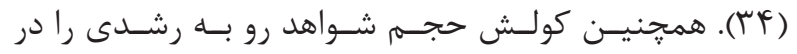

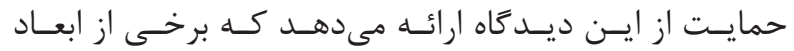

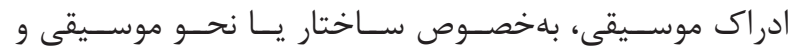

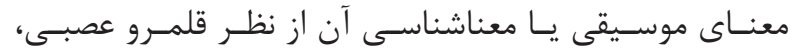

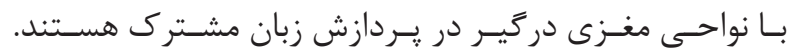

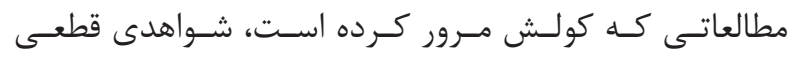

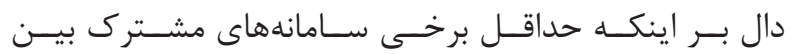

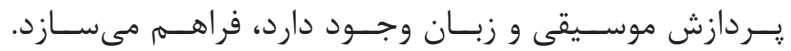

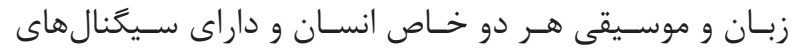

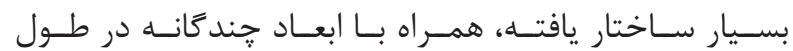

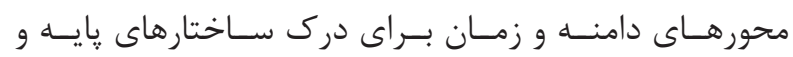

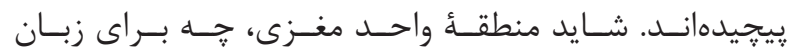

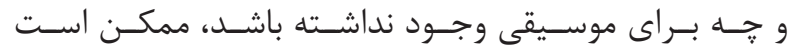

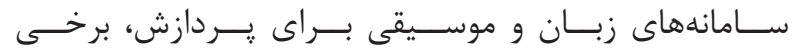

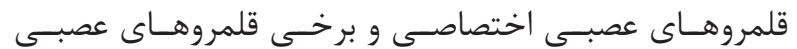

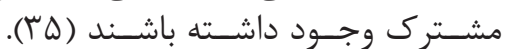

1. Miller GA. The science of words. New York: Scientific American Library. 1991.

2. Perani D, Saccuman MC, Scifo P, Spada D, Andreolli $\mathrm{G}$, Rovelli R, et al. Function specializations for music processing in the human newborn. Proc Natal Acad Sci. USA. 2010; 107(10): 4758-63.

3. Grodzinsky Y, Friederici AD. Neuroimaging of syntax and syntactic processing. Curr Opin Neurobiol. 2006; 16(2): 240-6.

4. Hagoort P. On broca, brain, and binding: a new framework. Trends Cogn Sci. 2005; 9(9): 416-23.

5. Eric Kandel JS, Jessell T. Principles of neural science the United States: McGraw-Hill Medical. 2000.

6. Standring S. Gray's anatomy: the anatomical basis of clinical practice. $39^{\text {th }}$ ed. Edinburgh: Churchill Livingstone. 2005.

7. Parker GJ, Luzzi S, Alexander DC, Wheeler-Kingshott CA, Ciccarelli O, Lambon Ralph MA. Lateralization of ventral and dorsal auditory-language pathways in the human brain. Neuroimaging. 2005; 24(3): 656-66.

8. Vigneau M, Beaucousin V, Hervé PY, Jobard G, Petit L, Crivello F, et al. What is right-hemisphere contribution to phonological, lexico-semantic, and sentence processing? Insights from a meta-analysis.

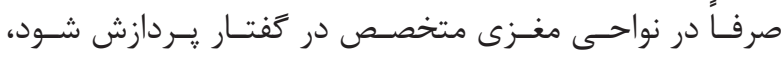

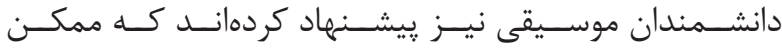

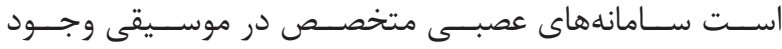

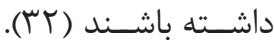

شــواهدى در حمايـت از سـامانههاى تخصصـى موســـــى در

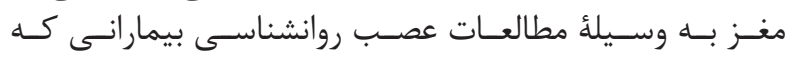

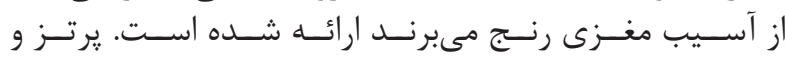

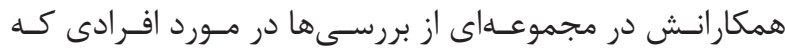

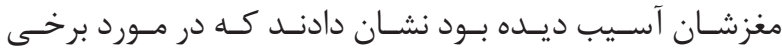

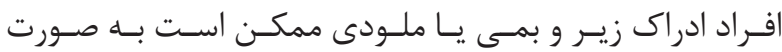

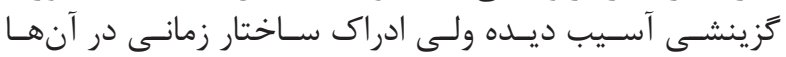

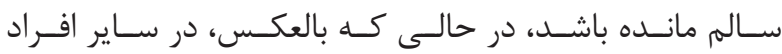

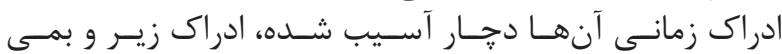
آنهـا سـالم باشــد (سانس)

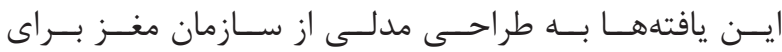

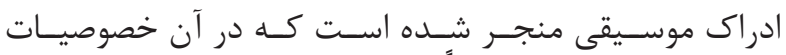

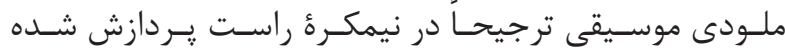

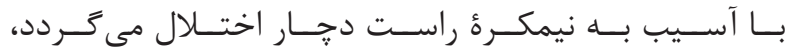

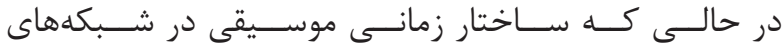

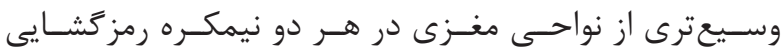

منابع

NeuroImage. 2010; 54(1): 577-93.

9. Zatorre RJ, Chen JL, Penhune VB. When the brain plays music: Auditory-motor interactions in music perception and production. Nat Rev Neurosci. 2007; 8(7): $547-58$.

10. Brown S, Martinez MJ, Parsons LM. Music and language side by side in the brain: A PET study of the generation of melodies and sentences. Eur J Neurosci. 2006; 23(10): 2791-803.

11. Brown S, Jordania J. Universals in the world's musics. Psychology of Music. 2011; 41(2): 229-48.

12. Buzsáki G. Rhythms of the brain. Oxford: Oxford University Press. 2006.

13. Sammler D, Koelsch S, Friederici AD. Are left frontotemporal brain areas a prerequisite for normal musicsyntactic processing? Cortex. 2011; 47(6): 659-73.

14 Liégeois-Chauvel C, Peretz I, Babaï M, Laguitton V, Chauvel P. Contribution of different cortical areas in the temporal lobes to music processing. Brain. 1998; 121(10): 1853-67.

15. Peretz I, Zatorre RJ. Brain organization for music processing. Annu Rev Psychol. 2005; 56: 89-114.

16. Chen JL, Penhune VB, Zatorre RJ. Listening to 


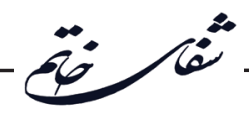

musical rhythms recruits motor regions of the brain. Cereb Cortex. 2008; 18(12): 2844-54.

17. Sammler D, Grigutsch M, Fritz T, Koelsch S. Music and emotion: electrophysiological correlates of the processing of pleasant and unpleasant music. Psychophysiology. 2007; 44(2): 293-304.

18. Milner B. Laterality effects in audition. Mountcastle VB. Interhemispheric relations and cerebral dominance. Baltimore: John Hopkins University Press. 1962; p. 177-95.

19. Nilipour R. Linguistic and pathology of language. Hermes Publication. 2002.

20. Sleeper AA. Speech and language. the United States of America: Chelsea House. 2007.

21. Binder J. The new neuroanatomy of speech perception. Brain. 2000; 123: 2371-2.

22. Zatorre RJ. Absolute pitch: a paradigm for understanding the influence of genes and development on cognitive function. Nat Neurosci. 2003; 6(7): 692-5.

23. Stewart L, von Kriegstein K, Warren JD, Griffiths TD. Griffi ths. music and the brain: disorders of musical listening. Brain. 2006; 129(10): 2533-53.

24. Koelsch S, Siebel WA. Towards a neural basis of music perception. Trends Cogn. Sci. 2005; 9(12): 578-84.

25. Peretz I, Gagnon L, Hebert S, Macoir J. Singing in the brain: insights from cognitive neuropsychology. Music Percep. 2004; 21 :373-90.

26. Koelsch, S. Music-syntactic processing and auditory memory: Similarities and differences between ERAN and MMN. Psychophysiology. 2009; 46(1): 179-90.
27. Peretz I, Coltheart M. Modularity of music processing. Nat Neurosci. 2003; 6(7): 688-91.

28. Levitin DJ, Menon V. Musical structure is processed in "language" areas of the brain: a possible role for Brodmann Area 47 in temporal coherence. Neuroimage. 2003; 20(4): 2142-52.

29. Halpern AR, Zatorre RJ, Bouffard M, Johnson JA. Behavioral and neural correlates of perceived and imagined musical timbre. Neuropsychologia. 2004; 42(9): 1281-92.

30. Schlaug G. The brain of musicians: A model for functional and structural adaptation. Ann N Y Acad Sci. 2001; 930: 281-99.

31. Klein ME, Zatorre RJ. A role for the right superior temporal sulcus in categorical perception of musical chords. Neuropsychologia. 2011; 49(5): 878-87.

32. Koelsch S. Brain and music: a contribution to the investigation of central auditory processing with a new electro-physiological approach. Leipzig: Risse. 2000.

33. Peretz I, Gosselin N, Belin P, Zatorre RJ, Plailly J, Tillmann B. Music lexical networks: the cortical organization of music recognition. Ann N Y Acad Sci. 2009; 1169: 256-65.

34. Peretz I, Radeau M, Arguin M. Two-way interactions between music and language: Evidence from priming recognition of tune and lyrics in familiar songs. Mem Cognit. 2004; 32(1): 142-52.

35. Koelsch S, Kasper E, Sammler D, Schulze K, Gunter T, Friederici AD. Music, language and meaning: brain signatures of semantic processing. Nat Neurosci. 2004; 7(3): 302-7. 